\title{
Commissioning and Operation Status of Belle II and SuperKEKB
}

\section{Shoji Uno on behalf of the Belle II Collaboration}

High Energy Accelerator Research Organization (KEK), Institute of Particle and Nuclear Studies (IPNS) 1-1 Oho Tsukuba, Japan

E-mail: shoji.uno@kek.jp

The SuperKEKB accelerator and the Belle II detector are upgraded from the successful KEKB /Belle project. The first commissioning of SuperKEKB was done in 2016 without Belle II. Belle II was constructed separately and the roll-in was smoothly performed in April 2017. The first collision events were finally recorded in April 2018. The smaller collision spot was measured as compared with the bunch lengths of the beams. It indicates the nano-beam collision is really working. 


\section{Introduction}

The SuperKEKB accelerator and the Belle II detector are upgraded from the successful $\mathrm{KEKB} /$ Belle project. KEKB provided the world highest luminosity to Belle and the accumulated integrated luminosity reached around $1 \mathrm{ab}^{-1}$. Belle observed the $\mathrm{CP}$ violation in the neutral $B$ mesons using a huge data [1] and it was consistent with the prediction from the KM theory [2]. The target luminosity of SuperKEKB is higher than KEKB by factor 40 with new collision scheme (nano-beam collision schme). Belle II will accumulate $50 \mathrm{ab}^{-1}$ data and will search for physics beyond the Standard Model and will study the exotic particles like the four-quark state.

The first commissioning of SuperKEKB was performed for 5 months in 2016 without Belle II. Belle II was constructed in the pit separately and the roll-in was performed smoothly in April 2017. After that, the superconducting final focusing magnets were installed into the interaction region (IR) and the commissioning of new damping ring was carried out in the injection linac section. In the Belle II side, the global cosmic ray run (GCR) was carried out to check the DAQ/Trigger system and to collect the calibration data. The first collision data was finally recorded in April 2018. The intensive SuperKEKB accelerator tuning continues to obtain higher luminosity. Belle II has been accumulating the collision data time to time and several known particles were reconstructed using the charged tracks and the photon clusters. The smaller collision spot was measured as compared with the bunch lengths of the beams. It indicates the nano-beam collision scheme is working.

The commissioning of SuperKEKB and Belle II has started successfully, but it is just the start point for a long physics experiment.

\section{SuperKEKB}

The SuperKEKB accelerator is upgraded from KEKB as shown in Fig. 1. The target luminosity is $8 \times 10^{35} \mathrm{~cm}^{-2} \mathrm{~s}^{-1}$ and is higher than that of KEKB by factor 40 . The beam energies of the electrons in the High Energy Ring (HER) and the positrons in Low Energy Ring (LER) are $7 \mathrm{GeV}$ and $4 \mathrm{GeV}$, respectively. The energy of the LER increases to obtain larger dynamic acceptance. The designed HER and LER beam currents are 2.6 A and 3.6 A, respectively. Several upgrades were performed to achieve those performances. The most import point is to apply the nano-beam collision scheme. The lower emittance and the smaller vertical $\beta^{*}$ in the interaction point (IP) are critical. A new lattice design was applied for the HER and a totally new ring was constructed for the LER to obtain the low emittance. A pair of new superconductive final focusing magnets was designed and was fabricated. It is complicated and essential to squeeze the beams. There are quadrupole magnets, compensation solenoid magnets and correction magnets in the single cryostat. New TiN-coated beam pipes with the antechamber structure were designed and were constructed to reduce the photoelectron cloud in the LER. A new damping ring was constructed in the injection linac section to meet the request for the LER with the smaller acceptance. More detail for the SuperKEKB accelerator is described in the reference [3].

This paper describes the reason in the slightly different way why the nano-beam collision scheme is applied in SuperKEKB to achieve higher luminosity. The bunch luminosity is limited due to several reasons. The most serious one is the beam-beam tune shift limit in the $\mathrm{e}^{+} \mathrm{e}^{-}$collider and the beam blowup which occurs in higher bunch current. And higher bunch current and shorter 
bunch length might cause hardware troubles due to higher heat load of the higher order mode (HOM) loss. Therefore, the bunch luminosity in KEKB $\left(1.2 \times 10^{31} \mathrm{~cm}^{-2} \mathrm{~s}^{-1}\right)$ was not so high as compared with other accelerators. But, around 1600 bunches could be stored in each ring thanks to the double rings and the world highest luminosity was achieved. The storage of more bunches is relatively straightforward to get higher luminosity. However, number of bunches is limited to around 5000 in the rings due to the RF frequency and higher luminosity can be obtained by factor three only for the normal collision scheme. Fig. 2 shows the collision image for KEKB and SuperKEKB. The overlap region is rather large in KEKB even for the small crossing angle. The collision spot is much smaller in SuperKEKB thanks to the smaller horizontal beam size (lower emittance) and the larger crossing angle. In this case, each bunch can be treated as many subdivided bunches because each subdivided bunch collides on each subdivided bunch for the opposite bunch and the luminosity of each subdivided bunch can reach the limit of the bunch luminosity. Therefore, the nano-beam collision scheme is able to increase number of bunches significantly and to get the target luminosity.

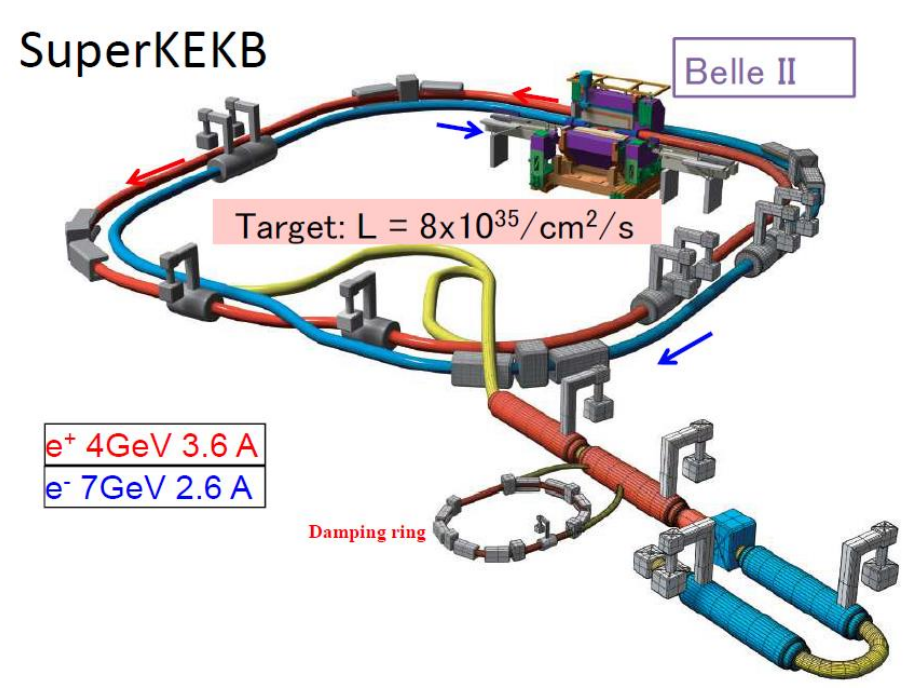

Figure 1: Schematic view of the SuperKEKB accelerator.

(a) KEKB

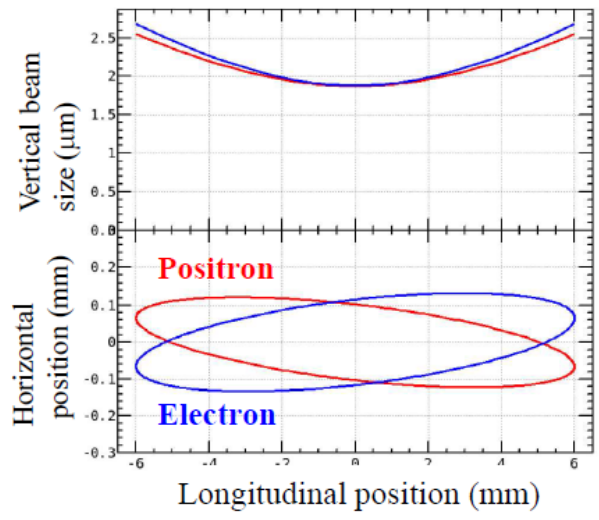

(b) SuperKEKB

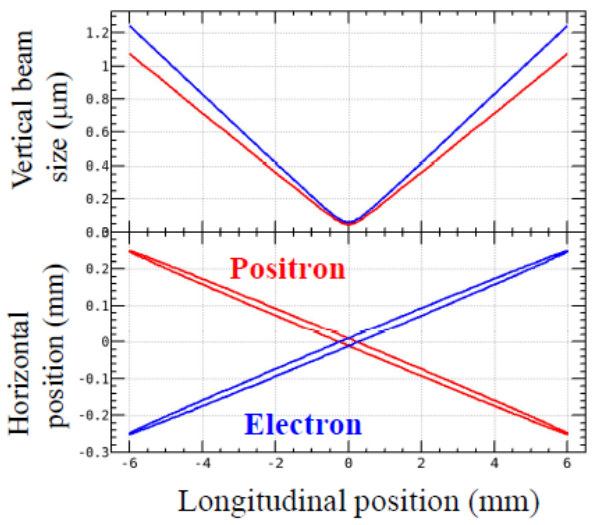

Figure 2: The vertical beam size and the collision image for both beams at KEKB (a) and SuperKEKB (b). 


\section{Belle II}

Larger beam background and higher trigger rate are expected due to higher luminosity in SuperKEKB. To accommodate such conditions, Belle II was upgraded from Belle with finer granularity in the detector segments, better timing separation and the pipeline readout scheme. Also, better performances are expected with new devices for the particle identification and the vertex measurement. Fig. 3 shows the Belle II detector, which consists of a new smaller beam pipe with $20 \mathrm{~mm}$ inner diameter, a new vertex detector (VXD; 2 layers DEPFET pixel detector and 4 layers double sized silicon strip detector), a 55-layers central drift chamber filled with He$\mathrm{C}_{2} \mathrm{H}_{6}$ gas mixture (CDC), the time of propagation Cherenkov counter using large quartz bars with MCP phototubes (TOP), the proximal focusing aerogel Cherenkov counter with HAPD photosensors(ARICH), reused $\mathrm{CsI}(\mathrm{Tl})$ calorimeters with new waveform sampling readout electronics both for the barrel and endcap regions (ECL) and reused resistive plate chambers for the barrel region and new scintillation counters including wave length shifting fibers with MPPC photosensors for the endcap regions and two inner layers of the barrel region (KLM). More detail of the Belle II detector is described in the design report [4].

The Belle II collaboration spreads out in the world. France and Israel joined recently. Number of collaborators reaches around 800 in 2018.

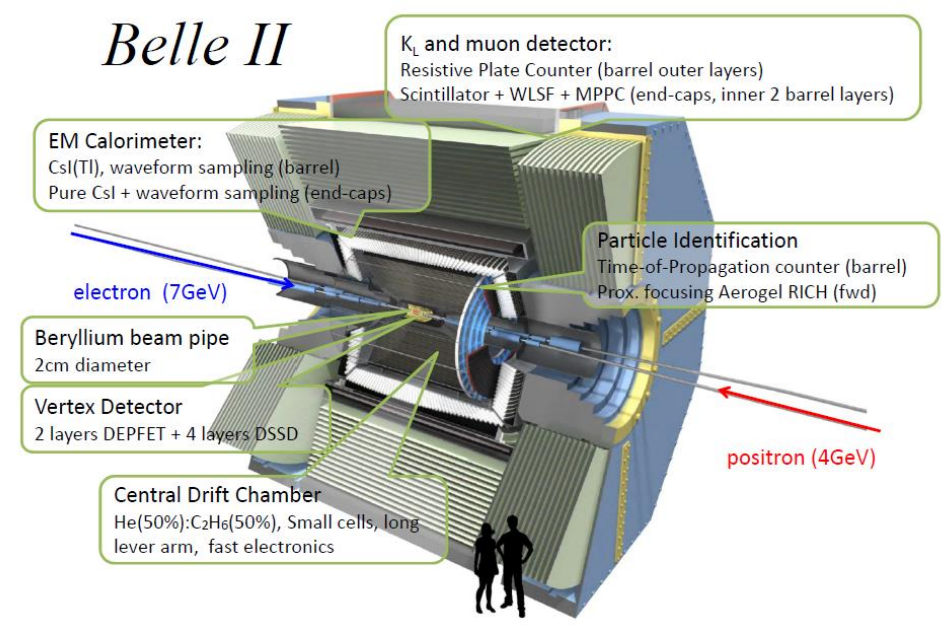

Figure 3: The Belle II detector.

\section{Commissioning}

There are three phases in the SuperKEKB and Belle II commissioning. The first commissioning of SuperKEKB was performed in 2016 without Belle II (Phase 1). The superconducting final focusing magnets were not installed at that time since the construction needed more time due to the complicated design. Also, the damping ring was not ready and was not necessary to inject the beam into the LER during the Phase 1 operation. The first beams were circulated at both rings in February 2016, successfully. At the beginning, the vacuum condition was quite bad even with low beam currents especially for the LER due to totally new beam pipes with the ante-chamber structure. The condition was much better for the HER, since the beam pipes are reused for most of parts. The baking condition could be kept in the construction period for several years. The stored beam currents increased gradually and reached around $1 \mathrm{~A}$ at the end of the Phase 1 operation. The optics correction and the response check of all quadrupole magnets 
have been carried out. The background monitor detector (BEAST II) consisting of several types of the background monitors was set in the IR without the solenoid field. BEAST II observed clearly the effect of the vacuum scrubbing and could extract the Touchek scattering effect from the beam gas related background. The observation indicated that the beam background is manageable for Belle II as compared with the Monte Carlo simulation. More detail of the result is shown in the reference [5].

After the Phase 1 operation, the superconducting final focusing magnets were installed and the commissioning of the damping ring was performed. Belle II was constructed in the pit and the roll-in was performed smoothly in April 2017 as shown in Fig. 4. After that, the global cosmic ray run (GCR) was carried out under $1.5 \mathrm{~T}$ magnetic field. Clear cosmic ray tracks were recorded for the installed outer detectors (CDC, TOP, ECL and KLM) with the central DAQ and trigger system. The data was used for the initial calibration and alignment for each detector. The remained detectors (ARICH, Forward endcap ECL and the beam background monitor including partial VXD) were installed after the GCR.

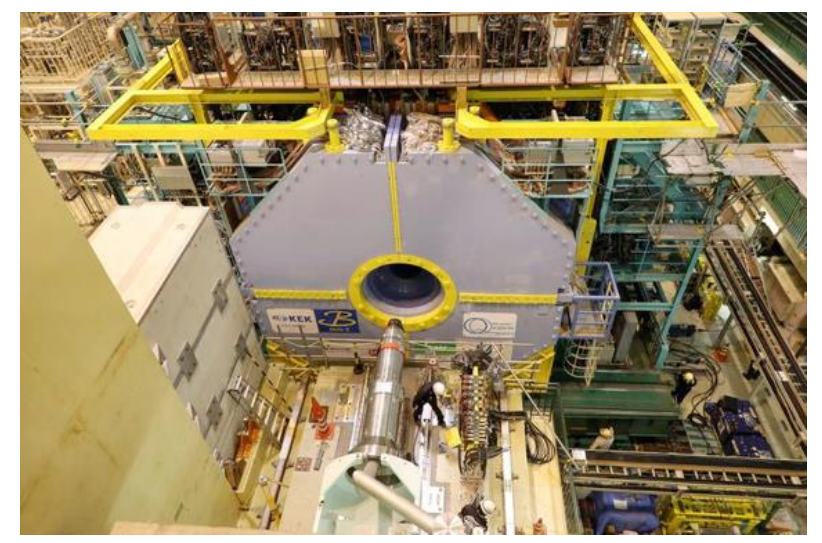

Figure 4: Phots of the Belle II detector just after the roll-in.

The first beams both in the HER and the LER were circulated through the Belle II IP chamber in March 2018, which was the start of the Phase 2 operation. The first collision events were observed with the Belle II detector in April after the intense beam tuning and the beam background study for a month. Fig.5 shows one of the hadronic events at the first collision. After that, the beams were squeezed step by step and the beam currents increased gradually while carefully monitoring the beam background in Belle II. SuperKEKB needs more time to obtain the target luminosity and to reduce the beam background. Belle II is still in the debugging and calibration stage. Even so, Belle II has recorded some amount of data. Several known particles were reconstructed with the correct mass positions and reasonable resolutions. Fig. 6 shows the $K_{s}^{0}$ mass peak for two charged tracks and Fig. 7 shows the $\pi^{0}$ mass peak for two photon clusters. Those plots indicates that the initial calibrations were already performed at reasonable level. The collision spot was also measured with the partial VXD with the CDC tracks. Fig. 8 shows the longitudinal size, which is $0.55 \mathrm{~mm}$ in the sigma and it is much smaller than the bunch length (around $6 \mathrm{~mm}$ ). It indicates that the nano-beam collision scheme is working.

After the Phase 2 operation (July in 2018), the full VXD will be installed and the Phase 3 operation will start in February 2019. 


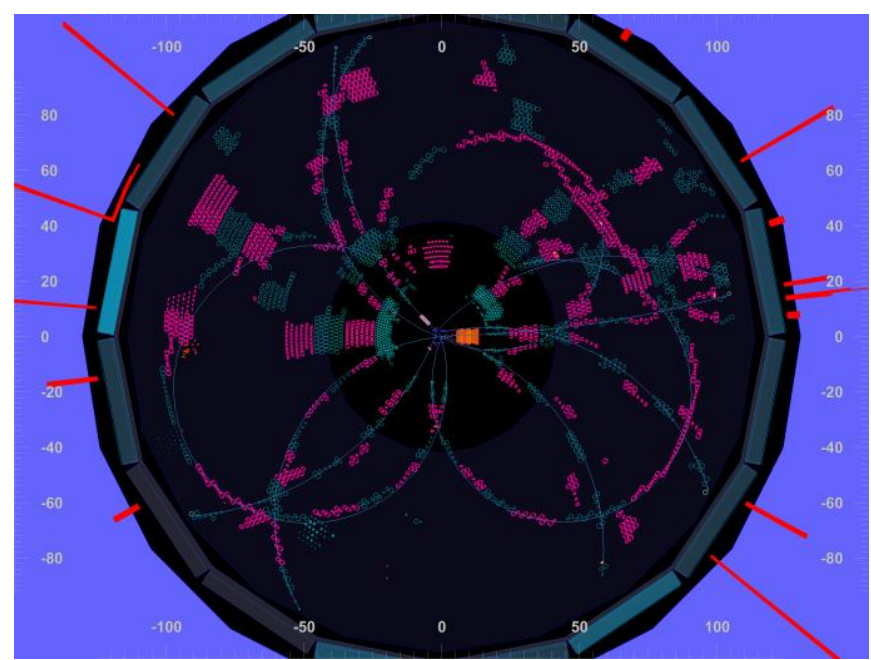

Figure 5: One of the first collision events

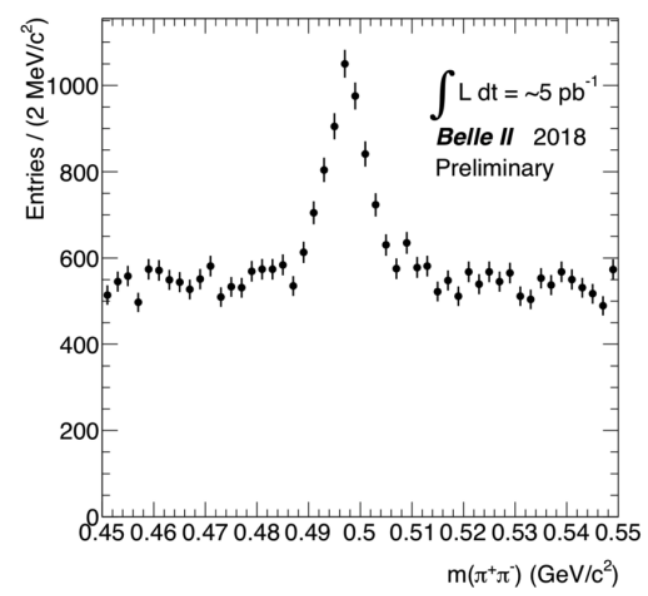

Figure 6: The $K_{s}^{0}$ mass distribution with two charged tracks.

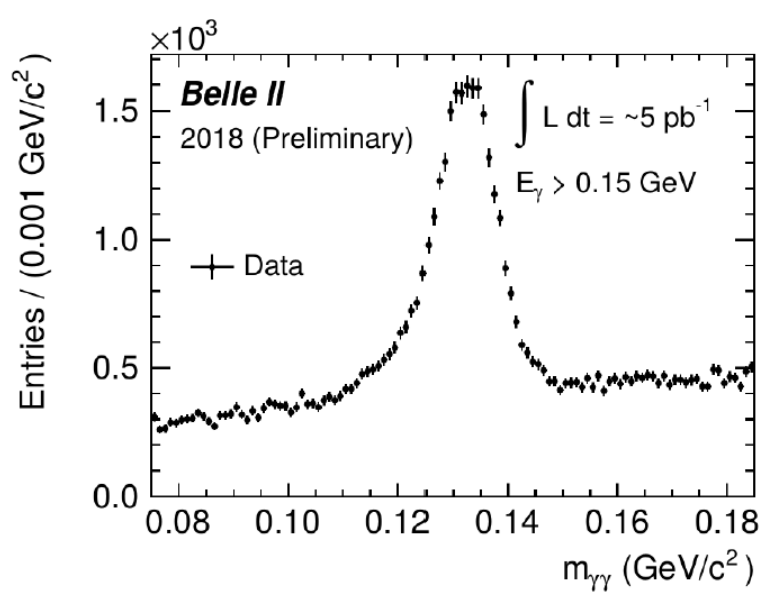

Figure 7: The $\pi^{0}$ mass distribution with two photon clusters. 


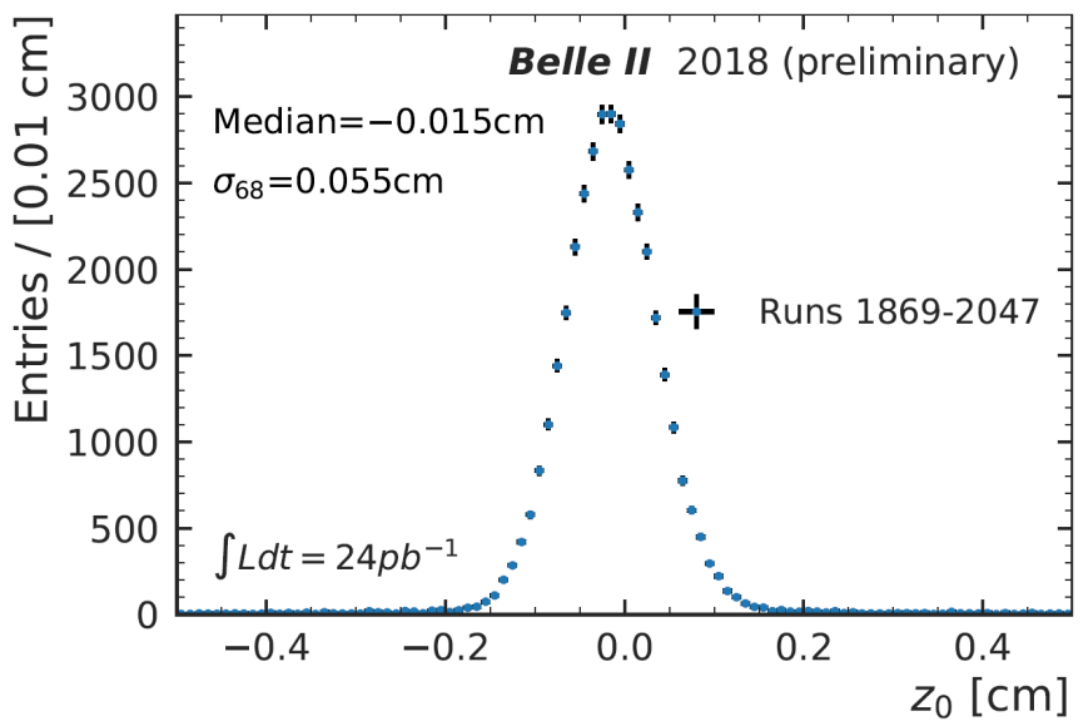

Figure 8: The longitudinal collision position measured with the VXD detector.

\section{Summary}

The SuperKEKB accelerator and the Belle II detector was constructed in several years after the end of the successful KEKB/Belle operation. The commissioning has been performed step by step. The first collision events were recorded with Belle II in April 2018. Reasonable mass distributions were obtained for known particles with charged tracks and photon clusters. Very smaller collision spot size was measured and it indicates that the nano-beam collision scheme is working.

\section{References}

[1] K. Abe et al., Belle Collaboration, Physical Review Letters 87, 091802-1 (2001).

[2] M. Kobayashi and T. Maskawa, Progress of Theoretical Physics 49, 652 (1973).

[3] Y. Ohnishi et al., Progress of Theoretical and Experimental Physics 2013 (3) (2013) 03 A011.

[4] T. Abe et al., Belle II Collaboration, arXiv:1011.0352 (2010).

[5] P. M. Lewis et al., arXiv:1802.01366 (2018). 